\title{
Artigo \\ Desigualdades sociais associadas com a letalidade por COVID-19 na cidade de Fortaleza, Ceará, 2020*
}

doi: 10.1590/51679-49742021000300022

\author{
Social inequalities associated with COVID-19 case fatality rate in Fortaleza, Ceará state, \\ Brazil, 2020
Desigualdades sociales asociadas con la letalidad por COVID-19 en la ciudad de Fortaleza, estado de Ceará, Brasil, 2020

\author{
Carlos Sanhueza-Sanzana' - (1) orcid.org/0000-0002-6021-564X \\ Italo Wesley Oliveira Aguiar ${ }^{1}$ - (1) orcid.org/0000-0002-7743-3109 \\ Rosa Lívia Freitas Almeida ${ }^{2}$ - (1) orcid.org/0000-0001-6423-543X \\ Carl Kendall $^{3}$ - (1) orcid.org/0000-0002-0794-4333 \\ Aminata Mendes' 1 - (1) orcid.org/0000-0001-6068-7682 \\ Ligia Regina Franco Sansigolo Kerr' ${ }^{1}$ - (0) orcid.org/0000-0003-4941-408X \\ 'Universidade Federal do Ceará, Programa de Pós-Graduação em Saúde Pública, Fortaleza, CE, Brasil \\ ¿Universidade de Fortaleza, Programa de Pós-Graduação em Saúde Coletiva, Fortaleza, CE, Brasil \\ ${ }^{3}$ Tulane University, School of Public Health and Tropical Medicine, Nova Orleans, LA, Estados Unidos
}

\section{Resumo}

Objetivo: Analisar a associação entre as desigualdades sociais e sanitárias, condições socioeconômicas, segregação espacial e letalidade por COVID-19 em Fortaleza, Ceará, Brasil. Métodos: Estudo ecológico de casos confirmados e óbitos por COVID-19, tendo como unidades de análise os 119 bairros de Fortaleza. Calcularam-se os indicacores de incidência, mortalidade e letalidade aparente por COVID-19, entre $1^{\circ}$ de janeiro e 8 de junho de 2020. Indicadores socioeconômicos foram extraídos do Censo Demográfico do Brasil de 2010. Foi realizada análise espacial e calculados índice global e local de Moran. Resultados: Foram encontrados 22.830 casos confirmados, 2.333 óbitos e uma letalidade aparente de 12,7\% ( $\left.\mathrm{IC}_{95 \%} 11,6 ; 13,9\right)$. Observaram-se autocorrelações espaciais significativas para letalidade aparente $(I=0,35)$ e extrema pobreza $(I=0,51)$, sobrepostas em diversos bairros da cidade. Conclusão: A letalidade aparente por COVID-19 está associada a piores condições socioeconômicas e de saúde, demonstrando a relação entre desigualdades sociais e desfechos de saúde em tempos de pandemia.

Palavras-chave: SARS-CoV-2; Fatores Socioeconômicos; Iniquidade Social; Letalidade; Mortalidade; Análise Espacial.

\footnotetext{
*Artigo derivado do projeto intitulado 'Desigualdades sociais na pandemia de COVID-19 em uma metrópole do Nordeste brasileiro: um estudo ecológico', idealizado pelo Grupo Cearense de Pesquisa em Doenças Infecciosas (GCPDI) do Programa de Pós-Graduação em Saúde Pública da Universidade Federal do Ceará em 2020.
}

\section{Endereço para correspondência:}

Carlos Sanhueza-Sanzana - Universidade Federal do Ceará, Faculdade de Medicina, Programa de Pos-Graduação em Saúde Pública, Rua Professor Costa Mendes, no 1608, $5^{\circ}$ andar, Rodolfo Teófilo, Fortaleza, CE, Brasil. CEP: 60430-140

E-mail: carlosanhueza.san@gmail.com 


\section{Introdução}

0 primeiro caso de pneumonia de origem não identificada foi notificado em Wuhan, a capital da província de Hubei, na China, em dezembro de 2019. 0 patógeno tem sido identificado como um novo RNA betacoronavírus, nomeado como síndrome respiratória aguda grave 2 (SARS-CoV-2), e a doença ocasionada por esse patógeno denominada coronavirus disease 2019 (COVID-19). ${ }^{1,2}$ Até 0 dia 4 de fevereiro de 2021, a infecção pelo vírus havia causado 2.260 .259 mortes em 103.989.900 casos confirmados em todo o mundo, ${ }^{3}$ registrando o Brasil o segundo lugar na carga de óbitos, 225.099, com 9.229.322 casos confirmados e 24.591 casos novos. $^{4}$

\section{Existem lacunas quanto ao conhecimento sobre as características de transmissão do vírus em um contexto de grande desigualdade social.}

A região Nordeste, uma das mais pobres do país, ${ }^{5}$ vem sendo profundamente afetada pela pandemia de COVID-19. Até o mesmo dia 4 de fevereiro, a região era responsável por $23,6 \%$ dos casos nacionais e apresentava a segunda maior incidência de novos casos: 526,7 por 100 mil habitantes. ${ }^{6} 0$ Ceará, um dos nove estados da região Nordeste, teve o primeiro caso notificado em 15 de março de 2020, com uma taxa de incidência da COVID-19 de 1.209,83 casos por 100 mil hab., sendo a segunda Unidade da Federação brasileira com o maior número de mortes acumuladas: 94,2 por cada 100 mil hab. ${ }^{4,7}$

Fortaleza, capital do Ceará, experimentou um aumento do número de óbitos, que ganhou velocidade e se estendeu até meados de maio de 2020, com um incremento exponencial à média diária de 36,6 óbitos. Fortaleza foi uma das capitais mais atingidas pela epidemia no país, depois das megametrópoles nacionais, como São Paulo e Rio de Janeiro. ${ }^{8}$

Análises retrospectivas de casos de síndrome respiratória aguda grave (severe acute respiratory syndrome, SARS), realizadas em laboratório, mostraram que o Ceará teve casos da COVID-19 no dia $1^{0}$ do mês de janeiro de 2020 , logo após o primeiro caso oficial ser notificado pelas autoridades de
Wuhan à Organização Mundial da Saúde (OMS). ${ }^{9,10}$ Disso se depreende que, quando o primeiro caso foi reconhecido, já existiam 1.303 casos notificados em Fortaleza e dois terços dos municípios do estado já apresentavam casos da COVID-19.

0 Brasil é um dos países mais desiguais do mundo em termos de concentração da riqueza. Essas desigualdades têm sido determinantes para o modo como a epidemia se disseminou nacionalmente, fazendo das regiões mais pobres, como o Norte e o Nordeste, aquelas com, respectivamente, a primeira e a segunda maiores prevalências de casos da COVID-19 no país.

A epidemia, tanto no Brasil como em diversas sociedades no mundo, revela com mais cor e nitidez os contrastes das desigualdades relacionadas ao gênero, ${ }^{11,12}$ status socioeconômico ${ }^{13}$ e raça ou etnia. ${ }^{14-16}$ De uma forma geral, no país, existem lacunas quanto ao conhecimento sobre as características de transmissão do vírus em um contexto de grande desigualdade social, com a população a viver em condições precárias de habitação e saneamento, sem acesso a água tratada e em situação de aglomeração. ${ }^{17}$

Visando conhecer em que medida as desigualdades sociais influenciam a saúde e a produção de riscos no contexto epidêmico, o objetivo desse estudo foi analisar a associação entre as desigualdades sociais por condições sanitárias, socioeconômicas, de segregação espacial e letalidade aparente por COVID-19 em bairros da cidade de Fortaleza.

\section{Métodos}

Foi realizado um estudo ecológico, cujas unidades de análises consistiram dos 119 bairros do município de Fortaleza. A capital do Ceará contava com uma população estimada de 2.669.342 hab. em $2020,{ }^{5}$ e a maior densidade demográfica entre todas as capitais brasileiras, 7.786,44 hab. por $\mathrm{km}^{2}$. Segundo dados do Censo Demográfico do Brasil de 2010, no que se refere à desigualdade social, a cidade apresentava coeficiente de Gini de 0,62 e grande proporção da população a viver em condições precárias, com $26 \%$ sem acesso a esgotamento sanitário adequado; havia um total de 508 favelas, onde residiam 396.370 pessoas, o equivalente a $16 \%$ da população total do município - 4\% superior ao percentual médio nacional., ${ }^{5,18}$

A população do estudo foi composta por todos os casos positivos e notificados de COVID-19. 
Os dados de notificação de casos e resultados de exames foram obtidos de repositórios de dados abertos, geridos pelo Governo do Estado do Ceará, referentes aos casos e óbitos notificados e confirmados da doença em Fortaleza, cujo banco de dados foi acessado no dia 9 de Junho de 2020 via plataforma de Integração das Informações da Secretaria da Saúde do Estado do Ceará. ${ }^{9}$ Foram incluídos dados de casos notificados entre os dias $1^{\circ}$ de janeiro e 8 de junho de 2020, de um total de 53.389 casos notificados no período, quando a mortalidade por COVID-19 no município atingiu seu primeiro pico epidêmico. ${ }^{9}$ Indicadores sociais e sanitários foram extraídos do Censo Demográfico do Brasil de 2010.

As variáveis do estudo contempladas foram:

a) Individuais

- sexo (feminino; masculino);

- faixa etária (em anos: menor que 1; 1 a 9; 10 a

19; 20 a 29; 30 a 39; 40 a 49; 50 a 59; 60 a 69; 70 ou mais);

- bairro de residência (pela denominação dos 119 bairros de Fortaleza).

Estas variáveis serviram à caracterização dos casos e óbitos analisados.

b) Agregadas por bairro

1) Indicadores epidemiológicos

- Número de casos confirmados por exame de transcrição reversa seguida de reação em cadeia por polimerase (reverse transcription polymerase chain reaction, RT-PCR) e número de óbitos por COVID-19. Os casos positivos confirmados referem-se aos casos que atendem à definição de caso preconizada pela OMS e Ministerio de Saúde: aqueles investigados epidemiologicamente e laboratorialmente, que confirmaram infecção pelo SARS-CoV-2 independentemente do critério de encerramento de caso (laboratorial, vínculo epidemiológico ou clínico);

- Número e tipo de comorbidades;

- Indicadores epidemiológicos calculados: (i) taxa de incidência de COVID-19, calculada pelo número de casos confirmados, entre $1^{\circ}$ de janeiro e 8 de junho de 2020, dividido pela população dos bairros multiplicada por 10 mil hab.; (ii) taxa de mortalidade específica por COVID-19, calculada para cada bairro pelo número de óbitos dividido pela população dos bairros e multiplicado por 10 mil habitantes; e (iii) taxa de letalidade aparente por COVID-19, estimada pela razão entre o número óbitos e o total de casos confirmados da doença.

2) Socioeconômicas e das condições sanitárias dos bairros

- Domicílio próprio; domicílios com acesso a esgotamento sanitário; domicílios com destino a fossa séptica; domicílios com 2 ou mais banheiros de uso exclusivo; domicílios com pessoa idosa (65 anos ou mais de idade), responsável por eles; domicílios com pessoas idosa ( $\geq 65$ anos) que não compartilham responsabilidades econômicas por ele, com qualquer outra pessoa; domicílios com pessoa acima de 15 anos de idade não alfabetizada; domicílios com renda mensal menor que 1 salário mínimo; e domicílios chefiados por mulheres;

- Mediana do rendimento familiar mensal per capita dos domicílios particulares, em reais (R\$); - Mediana de rendimento mensal de mulheres maiores de 10 anos de idade, com rendimento em reais $(\mathrm{R} \$)$.

3) Demográficas

- Domicílios com pessoas idosas ( $\geq 65$ anos); domicílios sem abastecimento de água da rede geral; domicílios sem coleta de lixo; domicílios sem acesso a energia elétrica; domicílios com mais de 4 moradores; e domicílios em situação de pobreza extrema;

- Taxa de mortalidade infantil $;$

- Mediana de moradores por domicílio.

- Indice de desenvolvimento humano por bairro (IDH-B), calculado pela Secretaria Municipal de Desenvolvimento Econômico (SDE) de Fortaleza; ${ }^{18}$

- Densidade demográfica (hab./ $\left./ \mathrm{km}^{2}\right)$.

4) Indicador de segregação espacial

Utilisou-se o índice socioeconômico do contexto geográfico para estudos em saúde (índice GeoSES), validado no Brasil. 0 índice se baseia nos dados do Censo Demográfico de 2010 para medir as desigualdades nas condições de saúde no nível territorial, em três escalas de agregação: nacional, federativa e intramunicipal (bairros). 0 GeoSES foi desenvolvido a partir de análises de componentes principais, a começar por 41 variáveis, resultando na definição de um escore com intervalos de -1 (pior situação socioeconômica) a 1 (melhor situação socioeconômica). 
0 GeoSES concebe o contexto socioeconômico da pessoa mediante avaliação de sete dimensões: educação; pobreza; riqueza; renda; segregação (critérios de educação e renda, concentrados nos extremos); mobilidade; e privação de recursos e serviços. ${ }^{19}$

Realizou-se a estatística descritiva das frequências relativas das variáveis individuais dos casos e óbitos confirmados por COVID-19, segundo sexo, faixa etária e comorbidades presentes, para o período em que foi realizado o estudo. 0s dados numéricos foram expressos como média e desvio-padrão (Dp), e, na ausência da distribuição normal, como mediana e intervalo interquartil de $25^{\circ}$ a $75^{\circ}$. A normalidade da distribuição das variáveis de tipo numérica foi avaliada pelo teste de Shapiro Wilk. Os dados de contagem foram expressos como casos e percentagens, apresentados com os intervalos de confiança. Utilizou-se o índice de Moran (I) como medida de autocorrelação espacial, para identificar a existência ou não de um padrão espacialmente condicionado pelos indicadores epdemiológicos de letalidade aparente, mortalidade específica e incidência de COVID-19, e os indicadores socioeconômicos, condições sanitárias, sociodemográficas e de segregação espacial. Utilizou-se a matriz de vizinhança queen de contiguidade de primeira ordem, a um padrão espacial significativo $<5 \%$. Adotaram-se, ainda, indicadores locais de associação espacial (local indicators of spatial association, LISA), para identificar aglomerados no município e suas significâncias estatísticas, com representação gráfica pelo LisaMap. Foram definidos e assim apresentados clusters - alto-alto; baixo-baixo; alto-baixo; baixo-alto - na forma de gráficos de dispersão (scatterplot) para as variáveis socioeconômicas, sociodemográficas e de segregação espacial que resultaram estatísticamente significativas, $<5 \%$.

A organização, limpeza e análises descritivas dos dados foi realizada por meio do software Stata versão 16. Os softwares Quantum Geopraphic Information System (QGIS) versão 3.12.1 e GeoDa versão 1.14.0 foram utilizados para as análises espaciais.

\section{Resultados}

Entre o dia $1^{\circ}$ de janeiro, data de registro do primeiro caso confirmado por COVID-19, até o dia 8 de junho de 2020 , foram observados 22.830 casos confirmados e 2.333 óbitos pela doença na cidade de Fortaleza.
Observou-se maior proporção de casos confirmados em mulheres $(54,0 \%)$; e de óbitos, entre os homens $(57,4 \%)$ (Tabela 1$)$.

Grande parte dos casos confirmados de COVID-19 encontravam-se na faixa etária de 40 a 59 (35,1\%), seguida dos 30 aos 39 (21,9\%) e daqueles com 70 ou mais anos de idade $(15,7 \%)$.

As mortes por COVID-19 concentraram-se na faixa acima de 60 anos, que representou aproximados $75 \%$ do total de óbitos, seguida pela de 40 a 59 anos (19,8\%). Também se observou uma quantidade menor de óbitos na idade inferior a 19 anos, representando $0,5 \%$ do total. Entre os casos confirmados, encontrou-se menor proporção de pessoas com comorbidades, como doença cardiovascular (5,3\%) e diabetes mellitus (4,7\%), comparadas aos óbitos, dos quais $34,9 \%$ tinham doença cardiovascular e, 31,9\%, diabetes. Destacou-se, também, a situação da saúde da mulher e da criança: Fortaleza registrou 25 casos de puérperas confirmadas com COVID-19, e destas, 4 vieram a óbito $(0,2 \%)$ (Tabela 1$)$.

0 município apresentou uma taxa de incidência de casos confirmados por COVID-19 de 66,4 ( IC $_{95 \%}$ $59,2 ; 73,6)$ por 10 mil hab. (Tabela 2), mais concentrada em bairros da zona norte e litoral da cidade, os mesmos bairros com piores condições socioeconômicas e sanitárias, a variar de 76 a 236 casos por 10 mil hab., seguidos de bairros da zona norte e zona sul, com 54 a 75 casos por 10 mil hab. (Figura 1A).

A cidade apresentou uma taxa de mortalidade específica por COVID-19 de 7,6 ( $\left.\mathrm{IC}_{95 \%} 6,8 ; 8,5\right)$ por 10 mil hab. (Tabela 2). Entre os bairros que registraram maior mortalidade, observou-se uma tendência a concentração naqueles da zona norte da cidade, com taxas a flutuar entre 6,8 e 26,5 óbitos por 10 mil hab. Chama a atenção a alta taxa de mortalidade nos limites da zona sul da cidade, região de altas vulnerabilidades sociais como pobreza, analfabetismo e baixa renda. Taxas de mortalidade muito mais baixas - inferiores a 6,7 por 10 mil hab. - foram identificadas nas áreas da cidade onde se encontrava a população com melhores rendimentos e condições sanitárias mais favoráveis (Figura 1B).

Fortaleza apresentou uma letalidade aparente por CoVID-19 de 12,7\% ( $\left.\mathrm{IC}_{95 \%} 11,6 ; 13,9\right)$, com uma média de casos confirmados por bair-

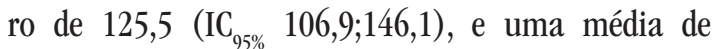
óbitos por bairro de 16,1 ( $\left.\mathrm{IC}_{95 \%}, 13,4 ; 18,6\right)$ (Tabela 2). 
Tabela 1 - Frequência absoluta e relativa de casos e óbitos confirmados por COVID-19, segundo sexo, faixa etária e comorbidades, Fortaleza, Ceará, 10 de janeiro a 8 de junho de 2020

\begin{tabular}{|c|c|c|c|c|}
\hline \multirow{2}{*}{ Variáveis } & Casos (n) & \multirow{2}{*}{ Casos (\%) } & Óbitos (n) & \multirow{2}{*}{ Óbitos (\%) } \\
\hline & $\mathrm{N}=22.830$ & & $\mathrm{~N}=2.333$ & \\
\hline \multicolumn{5}{|l|}{ Sexo } \\
\hline Feminino & 12.334 & 54,0 & 1.046 & 42,5 \\
\hline Masculino & 10.493 & 45,9 & 1.411 & 57,4 \\
\hline \multicolumn{5}{|c|}{ Faixa etária (anos) } \\
\hline$<1$ & 19 & 0,1 & 3 & 0,1 \\
\hline $1-9$ & 194 & 0,9 & 3 & 0,1 \\
\hline $10-19$ & 342 & 1,5 & 6 & 0,3 \\
\hline $20-29$ & 2.474 & 10,8 & 27 & 1,2 \\
\hline $30-39$ & 4.999 & 21,9 & 76 & 3,3 \\
\hline $40-59$ & 8.008 & 35,1 & 462 & 19,8 \\
\hline $60-69$ & 2.865 & 12,5 & 440 & 18,8 \\
\hline$\geq 70$ & 3.584 & 15,7 & 1.308 & 56,1 \\
\hline Não informado & 345 & 1,5 & 8 & 0,3 \\
\hline \multicolumn{5}{|l|}{ Comorbidades } \\
\hline Cardiovascular & 1.230 & 5,3 & 815 & 34,9 \\
\hline Diabetes mellitus & 1.089 & 4,7 & 799 & 31,9 \\
\hline Obesidade & 69 & 0,3 & 40 & 1,7 \\
\hline Renais & 161 & 0,7 & 105 & 4,5 \\
\hline Pneumopatias & 80 & 0,3 & 54 & 2,3 \\
\hline Neurológicas & 147 & 0,6 & 117 & 5,2 \\
\hline Imunodeficiências & 93 & 0,4 & 45 & 1,3 \\
\hline Puérperas & 25 & 0,1 & 4 & 0,2 \\
\hline Asma & 69 & 0,3 & 39 & 1,7 \\
\hline Hematológicas & 24 & 0,1 & 19 & 0,8 \\
\hline
\end{tabular}

Fonte: Secretaria da Saúde do Estado do Ceará, 2020.

Tabela 2 - Indicadores epidemiológicos de COVID-19 em bairros de Fortaleza, Ceará, $1^{\circ}$ de janeiro a 8 de junho de 2020

\begin{tabular}{|c|c|c|c|c|c|c|c|}
\hline \multirow{2}{*}{ Variável } & \multirow{2}{*}{ Média } & \multicolumn{2}{|c|}{$I_{95 \%}$} & \multirow{2}{*}{ Mediana } & \multirow{2}{*}{$\operatorname{lq} R$} & \multirow{2}{*}{ Desvio-padrão } & \multirow{2}{*}{ p-valor } \\
\hline & & LI & LS & & & & \\
\hline Taxa de incidência & 66,4 & 59,2 & 73,6 & 57,5 & 40,3 & 39,6 & $<0,01$ \\
\hline Taxa de mortalidade & 7,6 & 6,8 & 8,5 & 6,7 & 5,9 & 4,4 & $<0,01$ \\
\hline Taxa de letalidade aparente & 12,7 & 11,6 & 13,9 & 12,5 & 8,1 & 6,1 & 0,550 \\
\hline Óbitos & 16,1 & 13,4 & 18,6 & 12,0 & 18,0 & 14,3 & $<0,01$ \\
\hline Casos & 125,5 & 106,9 & 146,1 & 109,0 & 18,0 & 14,3 & $<0,01$ \\
\hline
\end{tabular}

Fonte: Secretaría da Saúde do Estado do Ceará, 2020.

a) Teste de distribuição normal Shapiro Wilk; $\mathrm{IC}_{95 \%}$ : intervalo de confiança de $95 \%$; LI: limite inferior do $\mathrm{IC}_{95 \%}$ da média; LS: limite superior do IC $\mathrm{C}_{95 \%}$ da média; IqR: intervalo interquartil. 


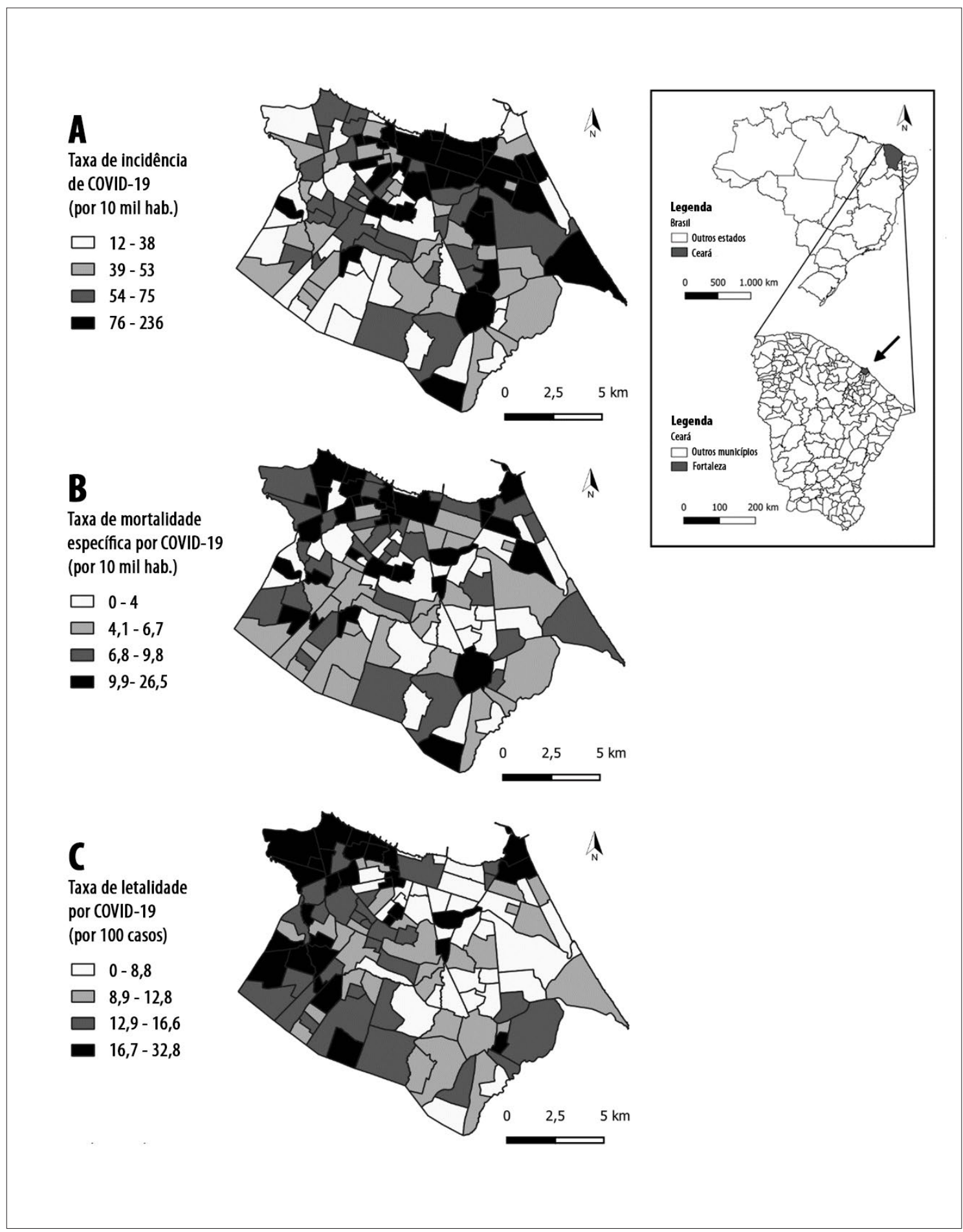

Fonte: Secretaria da Saúde do Estado do Ceará, 2020

Figura 1 - Distribuição espacial de óbitos, taxa de letalidade aparente e de mortalidade por COVID-19, Fortaleza, Ceará, $1^{10}$ de janeiro a 8 de junho de 2020 
Os bairros que apresentam maior letalidade aparente (Figura 1C) diferenciaram-se também como os de piores condições de vida; apresentaram (i) alto percentual de domicílios em condições de pobreza, acima de 39\% (Figura 2A), (ii) mediana de rendimento mensal baixa, até $\mathrm{R} \$ 600$ (Figura 2B), (iii) proporção maior de quantidade de domicílios chefiados por mulheres, entre $31,2 \%$ e 36,8\% (Figura 2C), (iv) menor proporção de pessoas na idade acima de 65 anos (Figura 2D) e (v) maior proporção de analfabetismo, entre 5,1\% e 11,7\% (Figuras 2E e 2F).

0 índice de segregação espacial GeoSES mostra que os bairros com maior letalidade também foram aqueles com maiores proporções de pobreza e piores condições de educação, renda e acesso a serviços de saúde, variando de -1 a -0,5 (Figura 2G).

A distribuição da letalidade por COVID-19 segundo os conglomerados LISA evidenciou a existência de bairros agregados alto-alto, estatisticamente significativos, na região noroeste da cidade ( $\mathrm{p}$-valor $<0,001$ ), e baixo-baixo em bairros localizados na região ao leste (p-valor<0,05) (Figura 3A). Quanto à proporção de domicílios em situação de pobreza, também se observaram agregados de bairros alto-alto na região noroeste e baixo-baixo na região leste (Figura 3B). Com relação ao índice de Moran, a letalidade apresentou um valor I de 0,35 (Figura 3E), e de 0,51 na avaliação por domicílios em condição de pobreza (Figura 3F).

\section{Discussão}

Os resultados evidenciam grande disparidade na distribuição dos óbitos por COVID-19 nos bairros de Fortaleza, demonstrando que a epidemia na cidade impacta desproporcionalmente, atingindo sobretudo as populações mais pobres. As características desiguais da distribuição e dispersão do SARS-CoV-2 na cidade de Fortaleza evidenciam uma estrutura desigual na exposição ao risco, fortemente associada à alta porcentagem da população excluída socialmente e segregada em áreas com precárias condições de vida. ${ }^{20}$ Destaca-se o fato da existência de uma dispersão progressiva da epidemia, de áreas com maior concentração de riqueza para bairros mais pobres. ${ }^{21}$

A segregação por classe socioeconômica e pela raça/ cor da pele é um forte determinante da saúde. Estudos têm demonstrado que comunidades afro-americanas altamente segregadas nos Estados Unidos sofrem de maiores taxas de mortalidade por efeito do vírus, ${ }^{15,22}$ achado igual ao de estudo realizado no município do Rio de Janeiro. ${ }^{23}$

Evidências com base em uma pesquisa populacional, realizada na cidade de Fortaleza no mês de junho de 2020, relataram uma soroprevalência de 14\% e uma estimativa de 370 mil pessoas tendo desenvolvido anticorpos para SARS-CoV- $2,{ }^{24}$ havendo uma alta população todavia suscetível à infecção pelo patógeno. De acordo com a mesma pesquisa, entre os bairros mais atingidos pela COVID-19, destacam-se os da região noroeste - Barra do Ceará, Pirambu e Cristo Redentor -, com prevalência de $20 \%, 3,5$ vezes superior à da população residente nos bairros de maior renda, evidenciando-se as disparidades em saúde, resultados que coincidem com os achados deste estudo, de alta letalidade aparente nesses bairros.

A letalidade aparente por COVID-19 reflete mais um padrão de estratificação social: a maior proporção dos domicílios são dirigidos por mulheres de baixa renda, das quais grande parte trabalha em áreas centrais da cidade, sob uma relação de emprego informal, com maior exposição ao vírus. Esse padrão poderia favorecer 0 transporte do vírus aos bairros mais empobrecidos, justificando a maior participação na taxa de letalidade aparente da população em situação de mais baixa renda - e maior pobreza - residente nesses bairros, proporcionalmente mais afetada.

A letalidade aparente por COVID-19 envolve também a assistência à saúde. Nas áreas com maior letalidade, observa-se maior dificuldade de acesso aos serviços de saúde de nível terciário (de maior complexidade), por conta da segregação espacial e da distância às unidades de pronto atendimento à saúde (UPAs).

0 caso brasileiro chama a atenção. As medidas políticas neoliberais adotadas agravaram os impactos da crise sanitária, tornando o Brasil o pior quadro da pandemia na América Latina. ${ }^{25-27}$ A experiência recente do país com outras epidemias - a exemplo da febre do chikungunya, febre Zika, dengue e febre amarela demonstrou o entrecruzamento dessas infecções com os indicadores demográficos, socioeconômicos e de saúde dos bolsões de pobreza e miséria, habitat de uma parcela expressiva da população. ${ }^{28}$

Nesse sentido, cabe considerar, entre as limitações do estudo, o fato de as variáveis demográficas e socioeconômicas utilizadas serem obtidas do Censo Demográfico de 2010, e, por isso, a possibilidade de não corresponderem à realidade de 2020. 


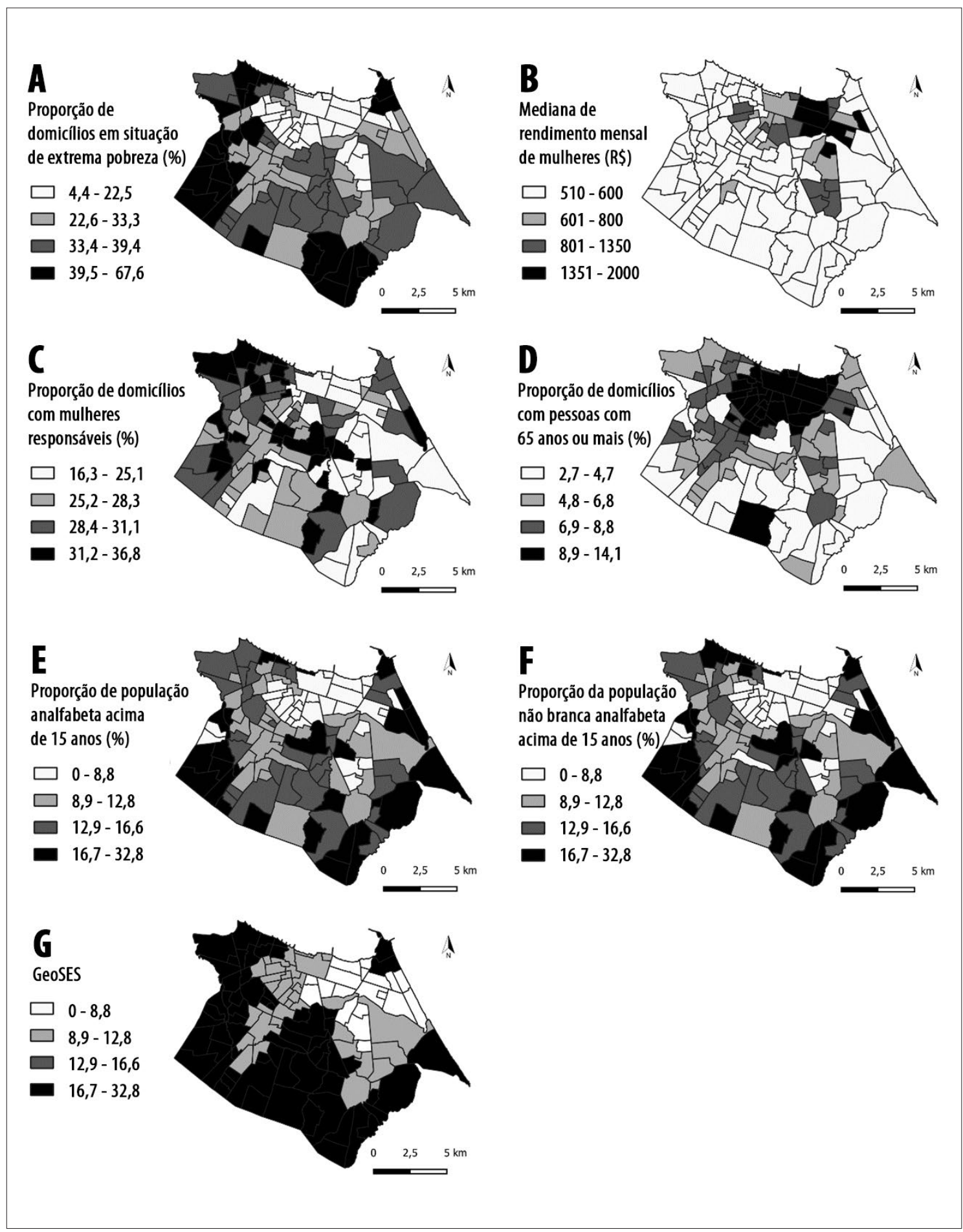

Fonte: Instituto Brasileiro de Geografia e Estatística (IBGE), Censo Demográfico 2010.

Figura 2 - Distribuição espacial dos indicadores socioeconômicos por bairros de Fortaleza, Ceará, 2010 


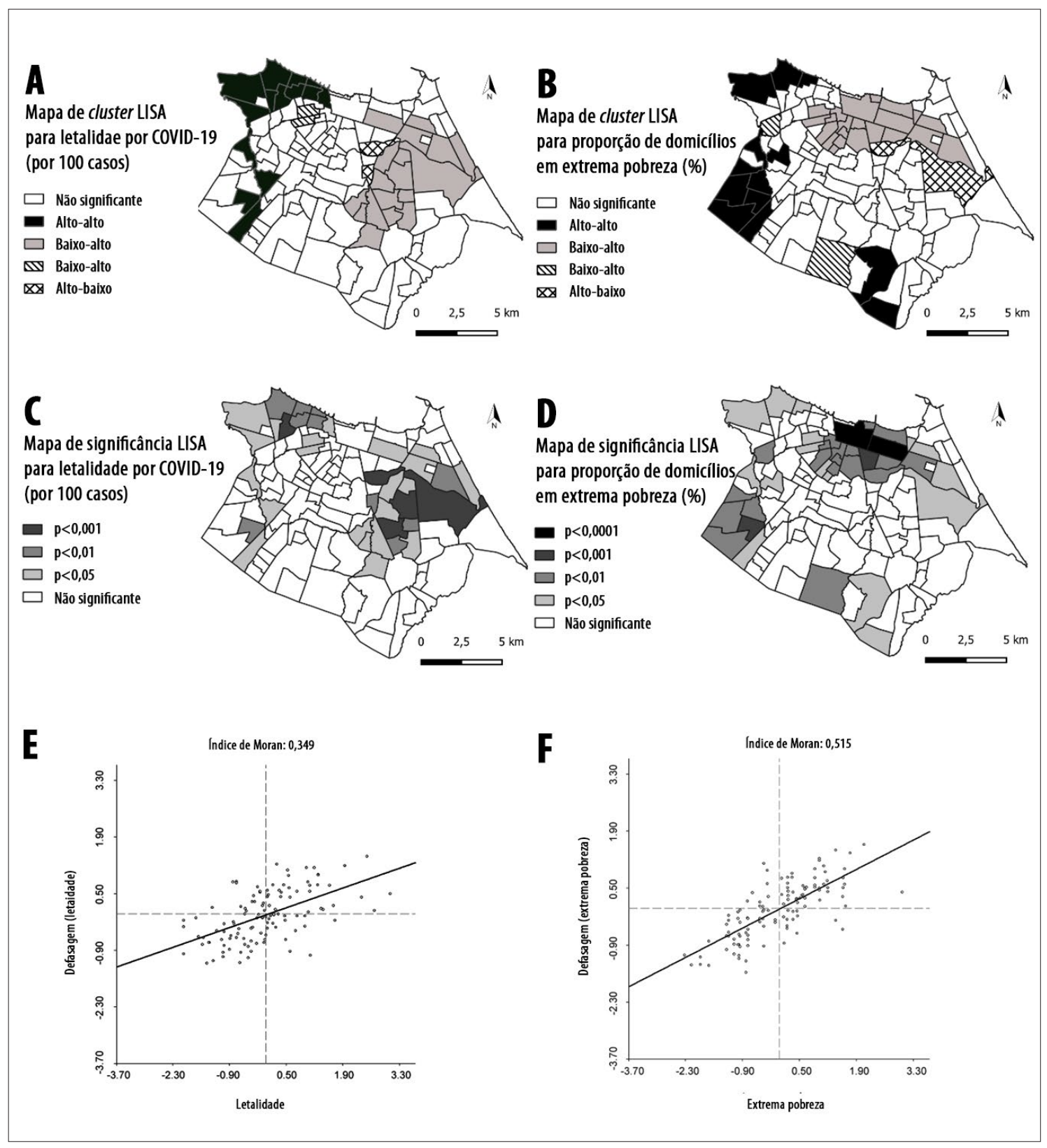

Fonte: Secretaria da Saúde do Estado do Ceará, 2020.

Figura 3 - Autocorrelação espacial da letalidade aparente por COVID-19 e extrema pobreza, segundo bairros de Fortaleza, Ceará, $1^{\circ}$ de janeiro a 8 de junho de 2020 
Entretanto, dados com o mesmo nível de desagregação e confiabilidade não estão disponíveis de forma atualizada, tendo em vista não se dispor de um Censo Demográfico do país mais recente.

Observa-se que bairros com alta letalidade por COVID-19 apresentam, também, deficiências nas condições sociais e econômicas, que se refletem em uma alta segregação espacial da população, associada a piores desfechos na doença. Tais condições impedem a aplicação bem-sucedida de métodos de prevenção. Recomenda-se que as populações dessas áreas sejam direcionadas para a testagem e rastreamento de contato, quarentena e distanciamento físico. No médio e no longo prazos, é crucial a implementação de políticas de melhoria das condições gerais de vida da população, promoção da saúde e prevenção de efeitos adversos de doenças infecciosas, emergentes e reemergentes.

0 estudo apresenta algumas limitações nos cálculos realizados para os indicadores epidemiológicos, haja vista uma quantidade considerável, e reconhecida,

\section{Referências}

1. Guan W-J, Ni Z-Y, Hu Y, Liang W-H, Ou C-Q, He J-X, et al. Clinical characteristics of coronavirus disease 2019 in China. N Engl J Med. 2020 Apr 30;382(18):170820. doi: https://doi.org/10.1056/NEJMoa2002032.

2. Li Q, Guan X, Wu P, Wang X, Zhou L, Tong Y, et al. Early transmission dynamics in Wuhan, China, of novel coronavirus-infected pneumonia. $\mathrm{N}$ Engl J Med. 2020 Mar 26;382(13):1199-207. doi: https://doi.org/10.1056/NEJMoa2001316.

3. World Health Organization. Situation by Region, country, territory \& area [Internet]; 2021 [acesso $4 \mathrm{fev}$. 2021]. Disponível em: https://covid19.who.int/table

4. Observatorio Fluminense. Covid-19 [Internet]. c2021 [acesso 4 fev. 2021]. Disponível em: https://www.covid19ri.org/

5. Instituto Brasileiro de Geografía e Estatística (BR). Sinopse do censo demografico 2010. Brasília, DF:IBGE: 2010 [acesso 3 jul. 2020]. Disponível em: https://biblioteca.ibge.gov.br/index.php/ biblioteca-catalogo?view $=$ detalhes\&id $=249230$ de subnotificação de casos novos e óbitos por COVID-19 no período da pandemia avaliado, além dos métodos empregados nesta análise. A demora na liberação de resultados e a restrita capacidade de testagem de casos para rastreio de indivíduos infectados, por parte dos serviços de saúde de Fortaleza, representa outro viés para o surgimento de indicadores diferentes do cenário real da pandemia na cidade.

\section{Contribuição dos autores}

Sanhueza CS e Aguiar IWO contribuíram no delineamento do estudo, análise e interpretação dos resultados, redação e revisão crítica do conteúdo do manuscrito. Kerr LRFS, Kendall C e Almeida RLF contribuíram na interpretação dos resultados e revisão crítica do conteúdo do manuscrito. Mendes A contribuiu na redação e revisão crítica do manuscrito. Todos os autores aprovaram a versão final do manuscrito e são responsáveis por todos os seus aspectos, incluindo a garantia de sua precisão e integridade.
6. Instituto de Comunicação e Informação Cientifica e Tecnológica em Saúde (BR). Monitoramento Covid-19 [Internet]. 2021 [ acesso 3 jul. 2020]. Disponível em: https://bigdata-covid19.icict.fiocruz.br/

7. Cunha Júnior A, Castalão DHS, Matos D, Basilio J, Livosolo L, Grave M, et al. Relatorio 8: progresso da Covid-19 no Brasil e no Estado do Rio de Janeiro: 26a semana epidemiológica [Internet]. Rio de Janeiro: Observatório Fluminense; 2020 [acesso 3 jul. 2020]. doi: https://doi. org/10.12957/eduerj.covid19rj.relatorio8

8. Ministerio da Saúde (BR), Secretaria de Vigilância Sanitária em Saúde, COE-COVID-19: semana epidemiológica 21 [Internet]. Brasília, DF: MS; 23 jun. 2020 [acesso 3 jul. 2020]. (Boletim Epidemiológico Especial, n. 17). Disponível em: https://antigo.saude.gov.br/images/pdf/2020/ May/29/2020-05-25---BEE17---Boletim-do-COE.pdf

9. Secretaria de Saúde (Ceará). Plataforma IntegraSUS: indicadores [sobre o novo Coronavirus (COVID-19)] [Internet]. [Ceará]: Secretaria 
de Saúde; 2020 [citado 3 jul. 2020]. Disponível em: https://integrasus.saude.ce.gov.br/

10. Candido DS, Claro IM, Jesus JG, Souza WM, Moreira FRR, Dellicour S, et al. Evolution and epidemic spread of SARS-CoV-2 in Brazil. Sience. 2020 Sep 4;369(6508):1255-60. doi: https://doi.org/10.1126/science.abd2161.

11. Wenham C, Smith J, Morgan R. COVID-19: the gendered impacts of the outbreak. Lancet. 2020;395(10227):8468. doi: https://doi.org/10.1016/S0140-6736(20)30526-2.

12. Alon T, Doepke M, Olmstead-Rumsey J, Tertil M. The impact of Covid-19 on gender equality. Cambrigde (MA): NMBER; 2020. (Working paper 26947). doi: https://doi.org/10.3386/w26947.

13. Chung RY-N, Dong D, Li MM. Socioeconomic gradient in health and the Covid-19. 2020 Apr 1;369:m1329. doi: http://dx.doi.org/doi:10.1136/bmj.m1329.

14. Goes EF, Ramos D0, Ferreira AJF. Desigualdades raciais em saúde e a pandemia da Covid-19. Trab Educ Saude. 2020;18(3):e00278110. doi: https://doi.org/10.1590/1981-7746-sol00278.

15. Kim SJ, Bostwick W. Social Vulnerability and Racial Inequality in Covid-19 Deaths in Chicago. Heal Educ Behav. 2020;47(4):509-13. doi: https:// doi.org/10.1177/1090198120929677.

16. Laurencin CT, McClinton A. The Covid-19 pandemic: a call to action to identify and address racial and ethnic disparities. J Racial Ethn Heal Disparities. 2020;7(3):398-402. doi: https:// doi.org/10.1007/s40615-020-00756-0.

17. Werneck GL, Carvalho MS. A pandemia de Covid-19 no Brasil : crônica de uma crise sanitária anunciada. Cad Saude Publica. 2020;36(5):e00068820. doi: http://dx.doi.org/10.1590/0102-311X00068820.

18. Prefeitura de Fortaleza (CE). Prefeitura apresenta estudo sobre desenvolvimento humano por bairro em Fortaleza [Internet]. Fortaleza (CE): SDE; 2011 [acesso 3 jul. 2020]. Disponível em: https:// pt.calameo.com/read/0032553521353dc27b3d9

19. Barrozo LV, Fornaciali M, André CDS, Morais GAZ, Mansur G, Cabral-Miranda W, et al. GeoSES: A socioeconomic index for health and social research in Brazil. PLoS One. 2020 Apr 29;15(4):e0232074. doi: https://doi.org/10.1371/journal.pone.0232074.

20. Instituto de Pesquisa e Estratégia Econômica do Ceará. Perfil municipal de Fortaleza: tema VIII: o mapa da extrema pobreza [Internet]. Fortaleza (CE): IPECE; out. 2012. [acesso 20 jul. 2020]. (Série infomre, n. 43). Disponível em: https://www.ipece. ce.gov.br/wp-content/uploads/sites/45/2012/12/ Ipece_Informe_43_05_novembro_2012.pdf

21. Ministerio da Saúde (BR). Doença pelo coronavírus Covid-19: Semana epidemiológica 24 [Internet]. Brasília, DF: MS; 2020 [acesso 20 jul. 2020]. Boletim epidemiológico especial, n. 18). Disponível em: http://antigo.saude.gov.br/images/pdf/2020/ June/18/Boletim-epidemiologico-COVID-2.pdf

22. Rentsch CT, Kidwai-Khan F, Tate JP, Park LS, King Jr. JT, Skanderson M, et al. Covid-19 by race and ethnicity: a national cohort study of 6 million United States veterans. medRxiv. Preprint 2020 May 17; doi: https://doi.org/10.1101/2020.05.12.20099135.

23. Cavalcante JR, Abreu AJL. Covid-19 no município Rio de Janeiro: distribuição espacial dos primeiros casos e óbitos confirmados. Epidemiol Serv Saude. 2020;29(3):e2020204. doi: https://doi. org/10.5123/S1679-49742020000300007.

24. Secretaria Municipal de Saúde. Inquérito soroprevalênca coronavírus em Fortaleza: dados preliminares [Internet]. Fortaleza (CE): SMS; 2020 [acesso 20 jul. 2020]. Disponível em: https://www.fortaleza.ce.gov.br/images/ COVID_INQU\%C3\%89RITO_19062020.pdf

25. Oliveira JF, Jorge DCP, Veiga RV, Rodrigues MS, Torquato MF, Silva NB, et al. Evaluating the burden of Covid-19 on hospital resources in Bahia, Brazil: a modelling-based analysis of 14.8 million individuals. medRxiv. Preprint 2020 . doi: https://doi.org/10.1101/2020.05.25.20105213.

26. Aquino EML, Silveira IH, Pescarini JM, Aquino $\mathrm{R}$, Souza-Filho JA. Rocha AS, et al. Medidas de distanciamento social no controle da pandemia de Covid-19: potenciais impactos e desafios no Brasil. Cien Saude Colet. 2020;25(suppl 1):2423-46. doi: https:// doi.org/10.1590/1413-81232020256.1.10502020.

27. Burki T. Covid-19 in Latin America. Lancet Infect Dis. 2020;20(5):547-8. doi: https://doi. org/10.1016/S1473-3099(20)30303-0.

28. Paixão ES, Teixeira MG, Rodrigues LC. Zika, chikungunya and dengue: the causes and threats of new and re-emerging arboviral diseases. BMC Glob Heath. 2018 Jan 4;3(Suppl 1):e000530. doi: https://doi.org/10.1136/bmjgh-2017-000530. 


\section{Abstract}

objective: To analyze the association among social and bealth inequalities, socioeconomic status, spatial segregation and Case Fatality Rate (CFR) due to COVID-19 in Fortaleza, the state capital of Ceará, Brazil. Methods: This was an ecological study of confirmed cases and deaths due to COVID-19. The 119 neighborhoods of Fortaleza were used as units of analysis. Incidence, mortality and apparent CFR indicators due to COVID-19 were calculated between January 1 and June 8, 2020. Socioeconomic indicators were obtained from the 2010 Brazilian Demographic Census. Spatial analysis was performed and local and global Moran's indexes were calculated. Results: There were 22,830 confirmed cases, 2,333 deaths and the apparent CFR was 12.7 (95\%CI 11.6;13.9). Significant spatial autocorrelations between apparent CFR ( $I=0.35)$ and extreme poverty ( $I=0.51)$, overlapping in several neighborboods of the city, were found. Conclusion: The apparent CFR due to COVID-19 is associated with the worst socioeconomic and health status, which shows the relationship between social inequalities and health outcomes in times of pandemic.

Keywords: SARS-CoV-2; Socioeconomic Factors; Social Inequity; Case Fatality Rate; Mortality; Spatial analysis.

\section{Resumen}

Objetivo: Analizar la asociación entre las desigualdades sociales y sanitarias, condiciones socioeconómicas, segregación espacial y letalidad por COVI-19 en Fortaleza, Ceará, Brasil. Métodos: Estudio ecológico de casos y defunciones confirmadas por COVID-19, se utilizaron, como unidades de análisis, 119 barrios de Fortaleza. Se calcularon los indicadores de incidencia, mortalidad y letalidad aparente por COVI-19, entre el 1 de enero y el 8 de junio de 2020. Los indicadores socioeconómicos se extrajeron del Censo Demográfico de Brasil 2010. Se realizó un análisis espacial y calculados los índices Global y Local de Moran. Resultados: Se encontraron 22.830 casos confirmados, 2.333 muertes y una letalidad aparente de 12,7 (IC $C_{95 \%}$ 11,6;13,9). Se observaron autocorrelaciones espaciales significativas para letalidad aparente $(I=0,35)$ y extrema pobreza $(I=0,51)$ que se sobreponen en diversos barrios de la ciudad. Conclusión: La letalidad por COVID-19 está asociada con peores condiciones socioeconómicas y sanitárias, demostrando la relación entre desigualdades sociales y los resultados de salud en tiempos de pandemia.

Palabras clave: SARS-CoV-2; Factores Socioeconómicos; Inequidad Social; Letalidad; Mortalidad; Análisis Espacial.

Recebido em 11/09/2020

Aprovado em 01/04/2021

Editora associada: Lúcia Rolim Santana de Freitas - (૯) orcid.org/0000-0003-0080-2858

Editora científica: Taís Freire Galvão - ( orcid.org/0000-0003-2072-4834

Editora geral: Leila Posenato Garcia - (1) orcid.org/0000-0003-1146-2641 